\title{
GLOBAL DESIGN OF AN ACTIVE INTEGRATED ANTENNA FOR MILLIMETER WAVE
}

\author{
Eric Marzolf, M'hamed Drissi \\ LCST/INSA de Rennes, 20 avenue des Buttes de Coësmes 35043 Rennes cedex, France \\ E-mail: Eric.Marzolf@insa-rennes.fr
}

\begin{abstract}
An active integrated antenna working in the millimeter wave has been realized in a monolithic process. The concept of active integrated antenna is first introduced, then the design of the integrated circuit based on a global approach, following electromagnetic and circuit simulations, is presented. The obtained performances of the active antenna are discussed and compared to a passive one.
\end{abstract}

\section{CONCEPT OF THE ACTIVE INTEGRATED ANTENNA}

Active integrated antennas (AIA) consist of integrating a radiating element with active circuits directly on the same substrate. A classification of such antenna modules which provide original architectures for modern microwave has been presented by T. Itoh in [1], based on the associated function. In a later review [2], the same author gives a deep insight of the benefits of the AIA concept: interesting properties are resulting by a tightly building of the radiation function and the signal processing.

Some recent developments show also that AIA can be applied in millimeter-wave systems [3]-[4], and that they prove to be an interesting concept in response to critical issues which exist in this bandwidth, as for instance losses in transmission lines or due to bulky interconnect between several radio parts. Fabrication technology for both $\mathrm{GaAs}$ and $\mathrm{Si}$ is maturing and makes them both attractive for AIA in the millimeter-wave band.

In this present work, we have associated an antenna to the input port of a low-noise amplifier on a same GaAs substrate. Hence, we obtain among others a greater antenna gain performance, a larger system bandwidth and a higher polarization purity. Also, as the antenna is considered as an integral part of the circuit, this means that the tight coupling between the different components has to be taken into account by an effort of a global simulation. As frequency increases, this is even more significant, so especially in the millimeter-wave band.

\section{DESIGN APPROACH}

The active integrated antenna (see figure 1) is realized in a HEMT technology, the ED02AH process from OMMIC, with a gate length of $0.2 \mu \mathrm{m}$ and a transition frequency of $63 \mathrm{GHz}$. The antenna consists of a circular patch fed by a simple microstrip line which connects directly to the input port of the amplifier. This keeps the purity of the radiated polarization at its best. It results that the radiated $\mathrm{E}$ plane is located in the same plane as the microstrip line. The amplifier has three transistor stages, all are polarized at the same bias point $(\mathrm{Vds}=3 \mathrm{~V}, \mathrm{Ids}=22 \mathrm{~mA})$. The obtained gain by the amplifier is of $11,2 \mathrm{~dB}$, its input is matched to the antenna impedance and its output to $50 \Omega$. 


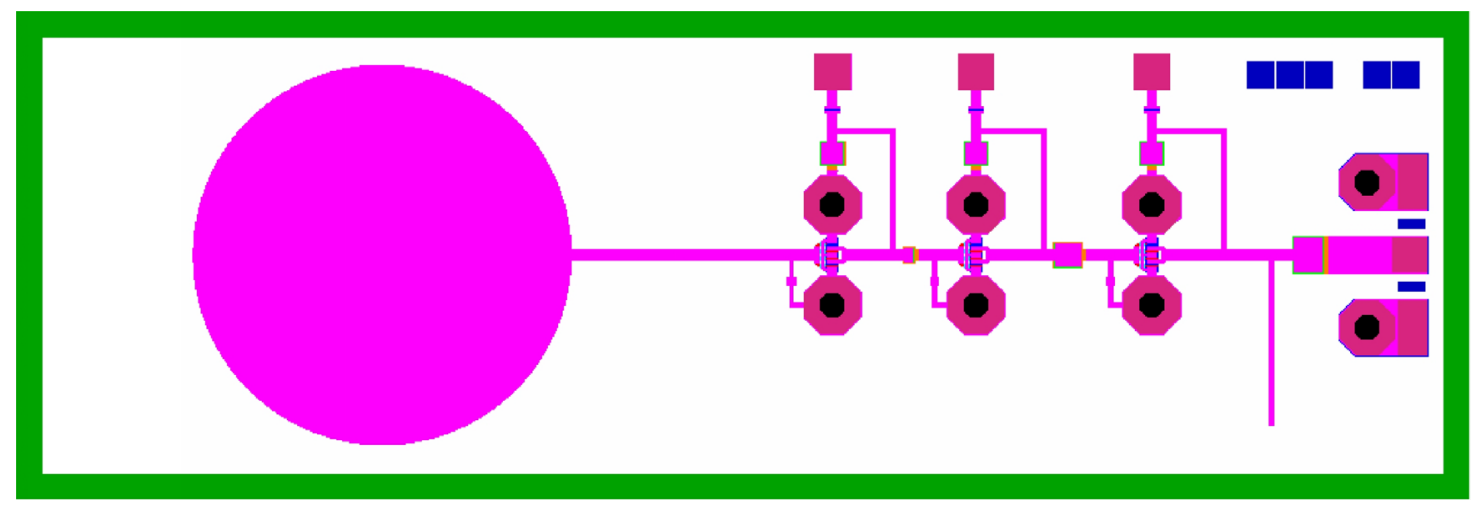

Figure 1: layout of the active integrated antenna

The circuit is designed by mean of a global approach, using a full wave analysis in the frequency domain [5], with the assumption that the corresponding network behaves linearly. An electromagnetic tool, based on the solving of the surface integral equations, is used to compute the compression matrix [6] resulting from the distributed parts of the circuit. Then a circuit analysis allows to connect the lumped elements together with the multi-port network, represented by the computed matrix. One should pay specifically attention to the problem of segmentation between the various parts of the circuit. Internal ports calibrated to $50 \Omega$ impedance are used to represent the interface with the lumped elements. External ports however, which remain calibrated to the characteristic impedance of corresponding transmission lines, are for a junction representation where no discontinuity appears. Finally all types of mutual coupling that exist in the circuit are rigorously taken into account, and matching between the different stages can better be handled.

\section{PERFORMANCES}

For the comparison of the results, a passive antenna is designed and matched to $50 \Omega$ as presented in figure 2.

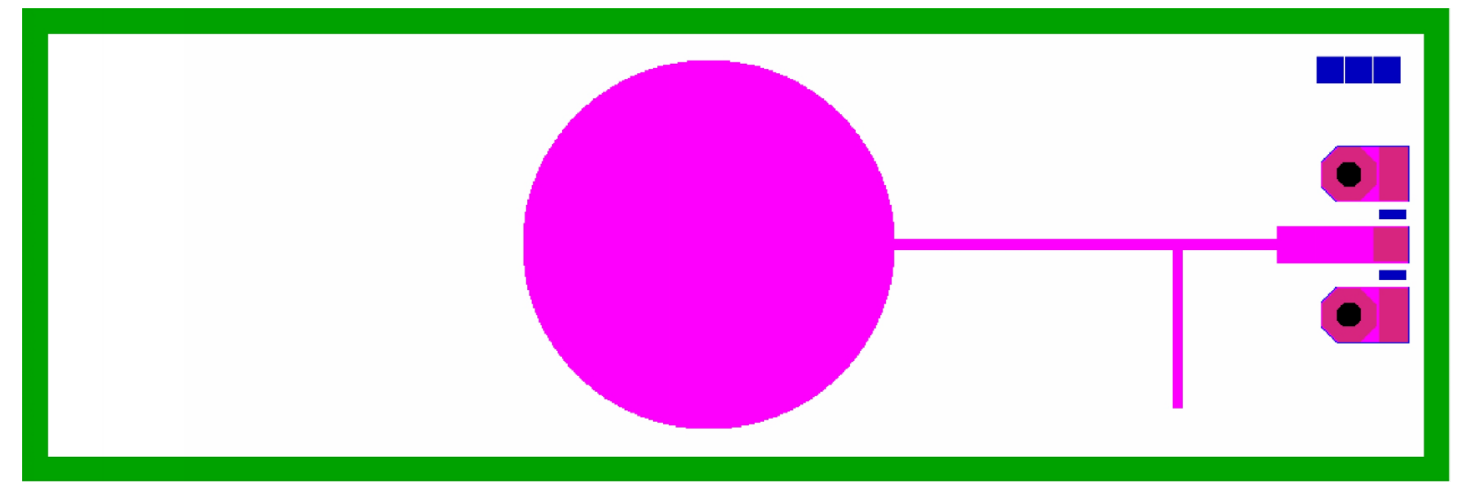

Figure 2: layout of the passive reference antenna

The S parameters, computed and measured are presented in figure 3. The measurements have been performed directly on wafer by mean of a coplanar probe station. The calibration has been done with standards realized on the same substrate. A phase shift is observed between simulation and measure of the passive antenna. For the active one, the bias point has shown to be somewhat different between simulation and measure. The best match has been obtained when the transistors were set to $(\mathrm{Vds}=1,6 \mathrm{~V}, \mathrm{Ids}=22 \mathrm{~mA})$. 


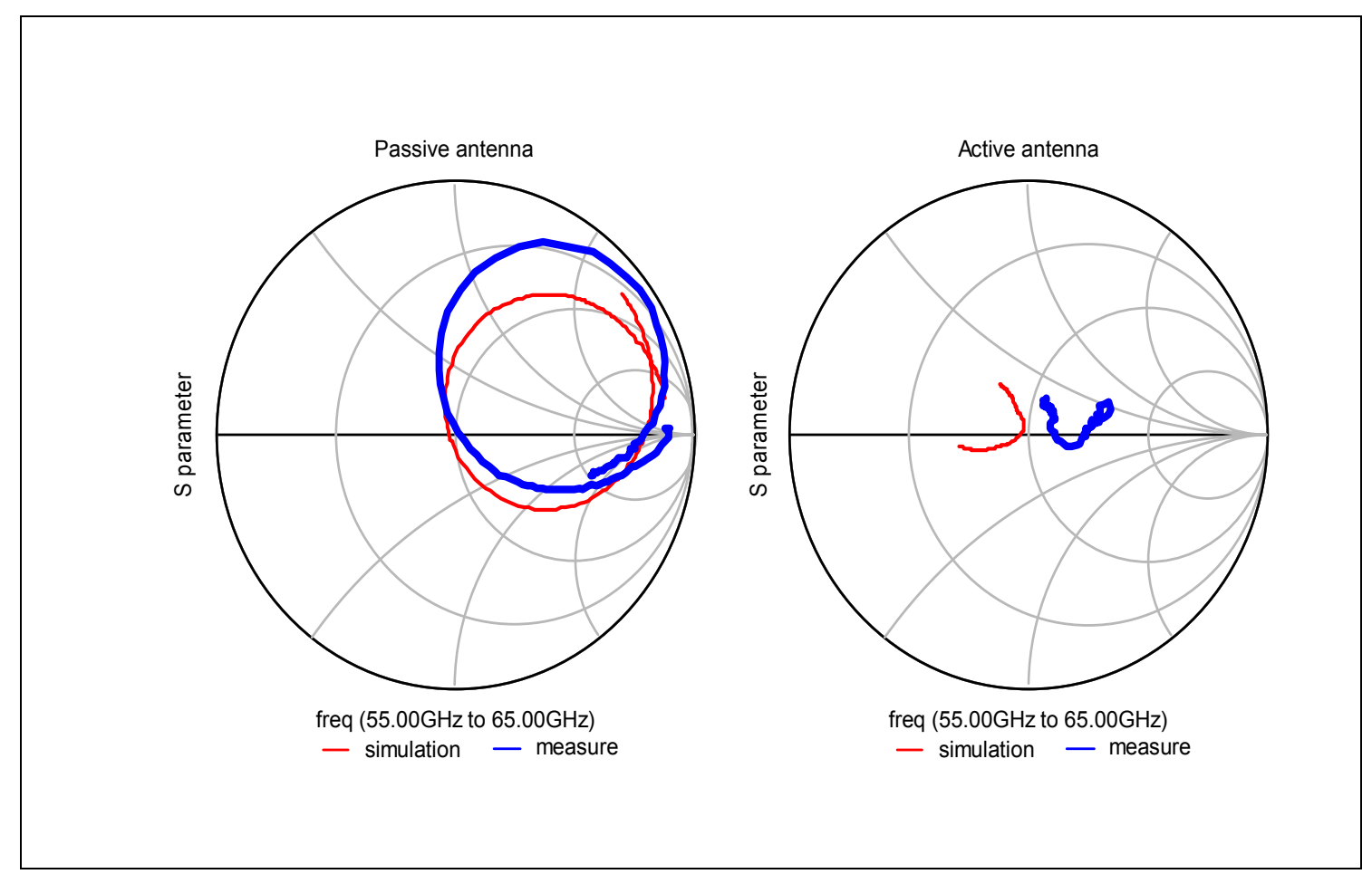

Figure 3: S parameter for the passive and the active antennas

Concerning the radiation performances, figure 4 shows the gain versus frequency for both structures. The antenna gain for the active structure is increased by the presence of the amplifier. On the other hand, the gain of the passive antenna matched to $50 \Omega$ is lower than the one corresponding to the circular patch alone. It is shown also by the figure 5 that the purity of the radiated polarization is optimal for the active structure. For the comparison, the loss of performance due to the matching network is showed for the passive structure.

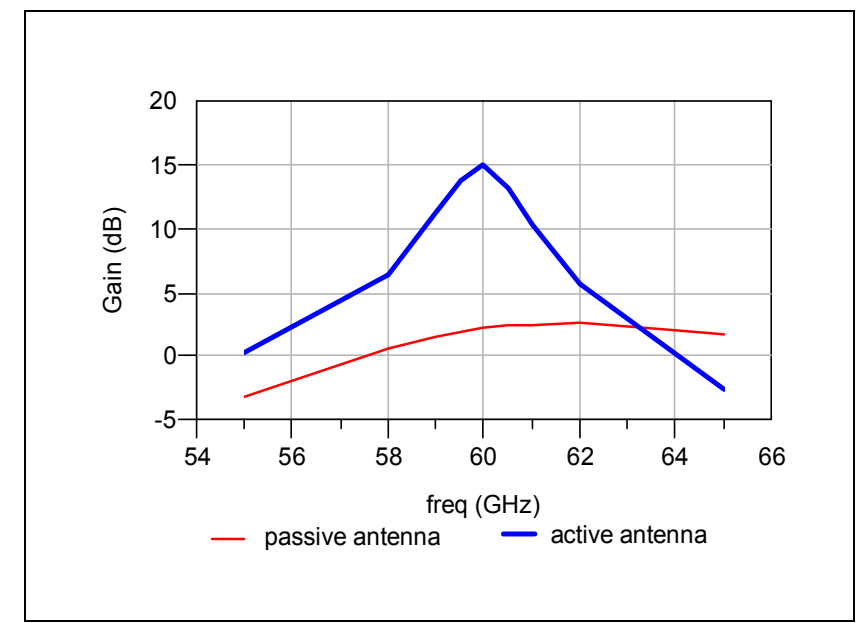

Figure 4: gain versus frequency for the passive and active antennas

\section{CONCLUSION}

It has been shown in this paper that in a receiver architecture the AIA concept improves the overall system performances like antenna gain (hence signal-to-noise ratio), working bandwidth and purity of radiated polarization over usual microstrip antennas. This makes them attractive for emerging applications like indoor broadband communications for millimeter-wave band. 
Finally, the global approach developed for the design of the active integrated antenna allows to treat the antenna as an integral part of the structure. Its increased complexity at millimeter wave, resulting from the various coupling, is also best modeled in this way.

\section{REFERENCES}

[1] J. LIN, T. ITOH, “Active integrated antennas”, IEEE Trans. MTT, vol. 42, pp. 2186-2194, 1994.

[2] Y. QIAN, T. ITOH, "Progress in active integrated antennas and their applications", IEEE Trans. MTT, vol. 46, pp. 1891-1900, 1998.

[3] E. M. BIEBL, "Millimeter wave systems based on active integrated antennas", Antennas and Propagation Millenium Conference, European Space Agency, Davos, 2000.

[4] Y. HIRACHI, S. KURODA, "Status of millimeter-wave MMIC's and their applications in Japan", European Microwave Conference, pp. 79-82, 2000.

[5] R. GILLARD, M. DRISSI, J. CITERNE, "Full wave analysis in the frequency domain", Analysis and design of integrated circuit antenna modules, edited by K. C. GUPTA and P. S. HALL, John Wiley, 2000.

[6] J. KUNISCH, M. RITTWEGER, S. HEINEN, I. WOLFF, "The compression approach : a new technique for the analysis of distributed circuits containing nonlinear elements", European Microwave Conference, pp. 1296-1301, 1991.
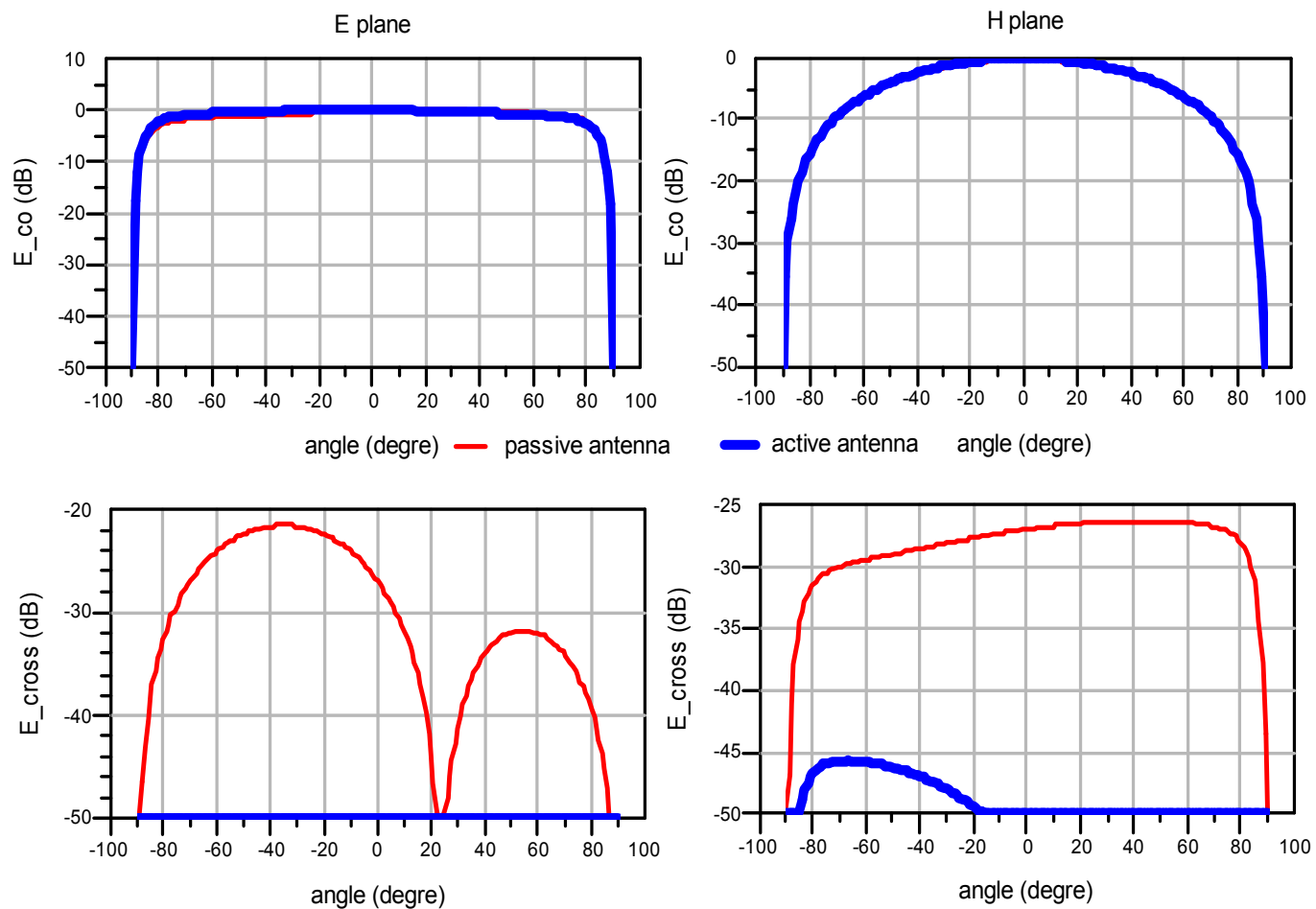

Figure 5: radiated field components for the passive and active antennas 